\title{
Model of Ground Handling Processes and Establishing Safety Mechanisms
}

\author{
Jindřich Duda ${ }^{1 *}$, Slobodan Stojić ${ }^{1}$
}

${ }^{1}$ Czech Technical University in Prague, Faculty of Transportation Sciences, Prague, Czech Republic

*Corresponding author: Czech Technical University in Prague, Faculty of Transportation Sciences, Horská 3, 12801 Prague, Czech Republic, Email: dudajind@fd.cvut.cz

\begin{abstract}
The following paper focuses on the operational safety issues in the aircraft ground handling process. Ground handling is a critical phase in terms of operational safety, however, severe injuries or even fatal accidents are rare. According to the available data, during a handling process a quite large number of incidents have as an outcome a damaged aircraft, which slows down the following processes and fluency of the relevant procedures. The financial costs in case of even the slightest damage are significantly high.

In order to implement different approach to safety management of ground handling companies, firstly a process analysis was performed using the Systems-Theoretic Accident Model and Process (STAMP) model. The STAMP model offers a different approach to operational safety as opposed to a traditional approach. STAMP approaches failure as a control error. The individual processes are designed for possible future STPA analysis.

The processes were modelled according to the publicly available sources, and further improved with the expertise and experience from a real operation. A list of potential deviations is added to the processes or individual activities. Coordination processes between the ground handling company and the airport were also discussed. An operational measure designed to increase operational safety is proposed for selected cases.
\end{abstract}

Keywords

Airport operations; ground handling; safety management; STAMP; modelling

\section{Introduction}

This article describes the issues of safety in the process of ground handling operation. These processes are carried out in a specific area of the airport. Usually, there is a large number of personnel and equipment around the aircraft, which are in some cases very close to the fuselage of the aircraft.

In this context, the aim of this paper is to show how the current approach to operational safety could be positively influenced. The airport, as the owner, provides its infrastructure to the external organizations operating at the airport. The airport should have at least a basic awareness of the processes taking place on this infrastructure. Such approach enables a creation of the general overview of the processes performed by the external organizations and provides strong foundations for their monitoring and management done by the airport.

The reason for this lays in a fact that airport is responsible for the operational safety within its premises, so it is interested in effective management and influencing of the safety related 
processes. As an effective tool in this approach the model STAMP [1] emerges as an interesting solution.

STAMP is a model that is used today in many industries and companies. STAMP enables easy description of the system's control structure and supports the idea of the establishment of the functioning control mechanism. It is created in a way, which enables monitoring and implementation of the safety measures on the spot that show irregularities. This is just one of the application of the created model, which is also extended through various tools developed on its basis such as tool STPA [2] (System-Theoretic Process analysis) used in the early phases of the system development for hazard analysis or STECA (System-Theoretic Early Concept Analysis) [3].

In this research the focus was placed on the ground handling processes, which are taking place at the airport. It tries to identify and manage the processes in which cooperation between the airports is crucial for flawless functioning of the system. Particular processes were modelled in the blockschematics, representing workflows with the clearly given responsibilities and roles. These models are prepared using the publically available documents dealing with the ground handling procedures and good practices, such as IATA manuals or Ground Operation Manuals of the individual airlines. It is important to highlight the fact that given processes are not adjusted to the specific conditions and environment of one existing airport, but were prepared as a globally accepted description of the ideal process, applicable anywhere.

Process models are considered as good starting materials for the new approach on which the potentially future analysis according to STPA tool could be easily performed without extensively deep knowledge of the investigated system. The whole approach is based on the defined deviations from the ideal process, which represent a dynamic list of potential irregularities, possibly leading to the safety event. The paper also discuss implementation of the possible safety measures based on a previously set deviation lists.

\section{Current approach to airport safety management}

The safety management system (SMS) provides framework and guidance for managing operational safety. The SMS manual [4] sets out the recommended minimum standard that shall be applied throughout companies functioning in the aviation industry. SMS is a systematic, precise and proactive process for managing safety risks. Full implementation will help proactively, reactively and predictively controlling risks to a level that is acceptable in the given conditions.

SMS is a content of Annex 19 [5] and The Doc. 9859 provides guidance material for the establishment of SMS requirements [4]. From the airport authority point of view, a ground handling company is one of the several subjects providing services at the airport. Having this situation, an airport has an intention to be familiar with the safety related processes of the external subjects.
Currently, airport has only passive approach to the management of the safety issues related to the external companies. This approach is given through the mechanism of the basic SMS elements:

1. Safety policy and goals

2. Risk management

3. Safety Assurance

4. Safety promotion

These however do not enable active role in the systematic approach to the process management. For those purposes, proposed approach supports creation of the high-level picture covering all coordination processes and sharing of the relevant data. This is a main reason, why this model was evaluated as adequate, taking into consideration its system-theory foundation and focus, which is placed on the definition of the system level hazards.

\section{Process modelling and following hazard analysis principles}

During the research phase, several processes were modelled according to their relevancy and priority. Firstly, the goal was to create a model of the current state, with all included entities, roles, responsibilities and control structure. Currently existing processes are now designed according to the principles of STAMP, however, internal control processes were analysed and used for the initial definition of the process deviations. The following Figure 1 show a process of approaching the aircraft, after full stop at the stand.

Individual process steps were described with the clearly stated activity, responsible entity and workflow. Each step was defined in a way that control loops from the STAMP model were specified for all of them. Failure of the particular control structure element is considered to be a deviation from the designed process.

On the previous figure 1, these deviation were defined for all process steps in a form of deviation list located next to the particular blocks in the schema. These represent detailed analysis of the process, which is basically a guideline for the safety expert performing an analysis or investigation. It is a question, whether these could be evaluated as hazards. It up to the safety expert on which level of detail will perform investigation and hazard analysis. STAMP and STPA take into consideration only system-level hazards.

In order to be in line with the STAMP and STPA principles, these deviation should be used as a control structure deviation descriptions and guidelines for extensive hazard identification.

\section{Internal processes coordination}

The airport has a specific structure with many sources of potential hazards. Therefore, monitoring of the ground handling 


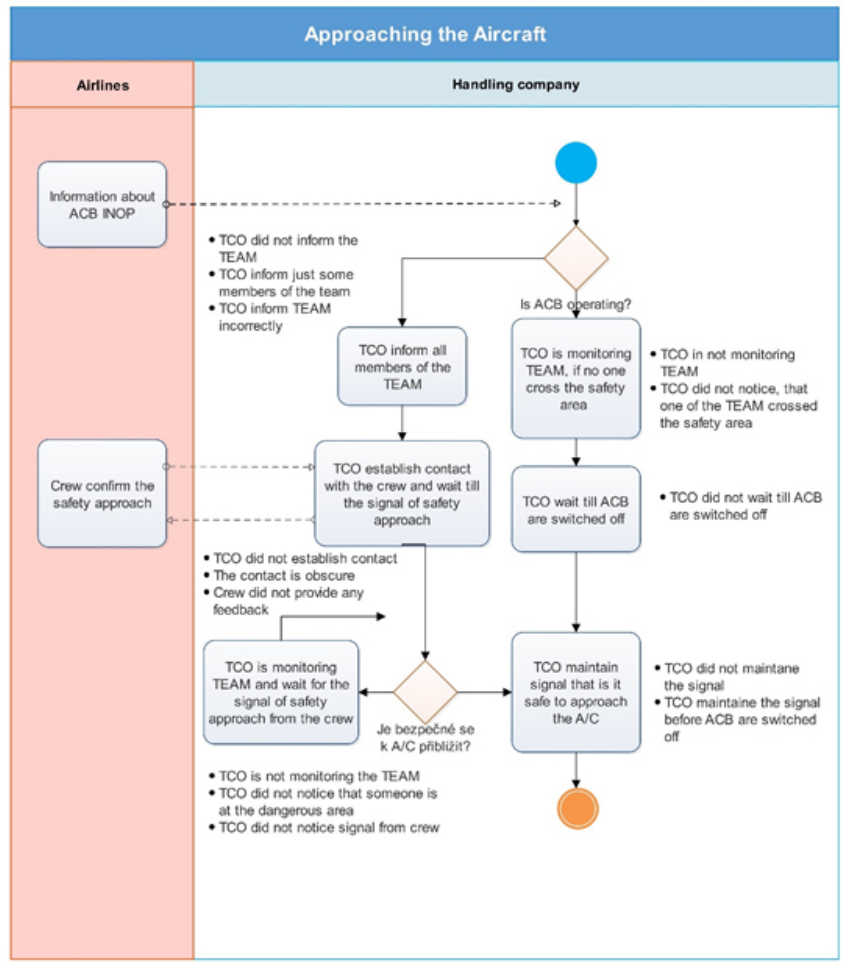

Figure 1. Model of a process - approaching the aircraft after full stop at the stand (source: author)

processes should be a very important part of the SMS. Thus, SMS is directly concerned with ground handling processes, given the number of potential hazards arising from them. The high level of safety culture and its promotion among employees has a major impact on successful safety management.

Having several organizations involved into one process brings a responsibility overlapping, which is not an exception in terms of safety. Airport and ground handling should have clearly given and coordinated safety mechanisms, functioning as one integrated end effective element. This integration and more proactive interconnections between organizations are represented in the following example. It is important to highlight, that coordination processes exists in the current systems, however, their utilization is only at the operational level.

\subsection{Process - Aircraft arrival}

After receiving an arrival assignment, the TCO will arrive at the assigned parking stand with a sufficient time to ensure that the stand is clear of all obstacles and equipment and that aircraft has unimpeded access to its final parking position. Prior to aircraft arrival, the team leader shall ensure that the following is available and serviceable at the arrival stand.

1. GSE

2. Chocks and Safety cones

3. Ground power source

4. Preconditioned air (if applicable)
Team leader must conduct pre-arrival briefing and allocate the assignments and responsibilities for the turnaround operation. This process should also ensure that all required staff are present and all required GSE is available and serviceable, positioned outside of ERA (Equipment Restraint Area). The team leader or designated person must ensure the crew involved in the operation understand and come to agreement with how the communication will be performed and how the aircraft will be handled.

The stand should also be checked for any FOD that is evident especially around the area to be occupied by the aircraft landing gears and engine intakes. Upon aircraft arrival, chocks and cones shall be positioned and the TCO is required to perform an aircraft arrival inspection for any aircraft damage. This shall be conducted prior to approach of any equipment, including all GSE and Passenger Boarding Bridge (Jetty). Minimum arrival aircraft inspection shall consist of the following:

1. All cargo doors

2. All access panels and servicing access points

3. Aircraft fuselage

4. Aircraft engine cowlings

\section{Aircraft passenger doors}

As an example here, a FOD removal will be used. The ramp agent, or other responsible person, performs the check, whether the aircraft stand is free of any obstacles or objects that could cause damage to the aircraft, while approaching the parking position. In the case of FOD detection, which could endanger a safe movement of the aircraft or the safe performance of the ground handling processes, the person in charge must remove the object outside the given area with a minimum delay.

If an obstacle is not possible to remove by the ground handling personnel or if a longer period of time is needed, or it is not the responsibility of the ground personnel to remove the FOD, and it is likely that the obstacle will not be removed prior to the arrival of the aircraft, ramp agent must inform the airport central operation dispatch, which will decide, if necessary, to allocate another airport stand for the flight. In this case, the central operation dispatch must inform the ground handling service provider immediately.

\section{Practical safety measures based on created safety model}

In a dynamic airport environment, where the change of arrival time or last-minute change of stand is done on daily basis, it is difficult for the ground handling organization to scheduled turn-around coordinators at the right time. And therefore, it is not always possible to reach the TCO with sufficient time to perform all the tasks before arrival of the aircraft. For practical 


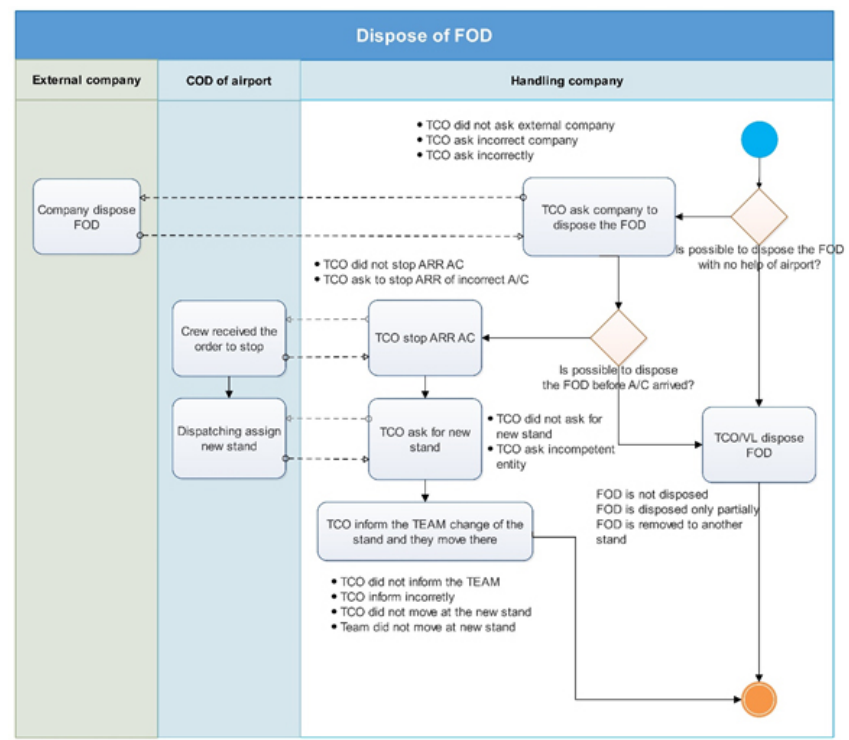

Figure 2. Model of the process - FOD removal (source: author)

presentation, one of the defined deviations could be analysed and safety measure explained.

One of the safety measures, with the high probability for process efficiency increase is a radar or scanner device, which provides automatic FOD check. Application of such device changes internal structure of the existing control mechanism through a reduction of the several control loops and implementation of the automated sensors and actuators.

The device in this context would represent integrated part of VDGS (visual docking guidance system). Its basic activity is started before the aircraft reach the stand. In case of late arrival of TCO on the stand and inability to perform FOD (Foreign object debris) check, the device is capable of FOD recognition and sharing of the positive finding message with the crew of an aircraft. The signal for the crew will be in a form of indication STOP on the display of VDGS.

Such system showed good results in revealing larger objects that are left on the stand, such as dollies, conveyor belts or other GSE (ground service equipment). From the practical stand point, one of the disadvantages that should be taken into considerations are technical issues for their utilization in a case of remote stands or other open aprons without supporting pylons or other fixed infrastructure. It would be also difficult to set up such sensitivity to reveal miniature objects, such as a screw or baggage lock.

\section{Limitations}

It should be highlighted that the process maps are not modelled for the particular airport or airline. The maps are based on IATA guidance materials and good practices [6, 7], which have defined the basic rules for the ground handling processes, in order to be performed safely, using current technology. Process maps can only be used as some kind of global back- ground (globally acceptable solution), but not as material corresponding with a particular airport conditions. The local conditions of a given airport, such as stand design, airport infrastructure or meteorological conditions, are not applied and should be implemented during system design phase of the process change. In case of the airlines, this organizations may have their specific requirements based on service experience, which may be different in comparison with IGOM [6].

\section{Conclusion}

The article describes particular ground handling processes through their modelling. For every modelled process step a list of deviations is defined. Deviation in this context is understood as a practical drift from the process as designed. These represent a guidelines for the investigation process in the systems, where no sufficient operational data are available and as a support for the preparation of the safety studies on the existing and new systems. Model respects STAMP principles and it is based on the control structure management.

Deviation in this context represent expected possible issues with the control mechanism, which is defined for all control loops. Due to a fact that this mechanism is defined on the existing, fully functioning system, this approach was evaluated as the most efficient. Potential optimization of the defined control structure is expected, after operational data analysis and evaluation of the acceptance of the system robustness and structure as it is given currently.

Analysis also highlighted the processes in which coordination between ground handling company and airport takes place. This is particularly important, because one of the basic principles of the STAMP is that only system level hazard should be a subject of hazard identification. In such complex systems, where responsibilities of the several entities overlap, it is important to establish functioning and effective management system with the system-level hazards identified and understood by all included parties.

Paper also brings a proposal of technological or process changes that would help to set up system and implement safety measures within the process. The process maps were defined with the intention of future use for complete STPA analysis. An analysis of possible measures based on a previously set hazard lists was performed according to the modelled processes. A validation method was recommended for each proposal.

\section{References}

[1] Nancy Leveson. Engineering a safer world : systems thinking applied to safety. The MIT Press, Cambridge, Mass, 2012. ISBN 9780262016629.

[2] Nancy Leveson and John Thomas. STPA Handbook. The MIT, 2018.

[3] Cody Fleming. Safety-driven Early Concept Analysis and Development. 2015. 
[4] ICAO. Doc 9859 Safety Management Manual. International Civil Aviation Organiyation, Montréal, Quebec, 2018. ISBN 978-92-9258-552-5.

[5] L-19 Letecký předpis řízení bezpečnosti, 2013. URL https://aim.rlp.cz/predpisy/predpisy / dokumenty/L/L-19/index.htm.

[6] IATA. IATA Ground Operations Manual (IGOM). International Air Transport Association, Montréal, Quebec, 2013. ISBN 978-92-9252-120-2.

[7] IATA. Airport Handling Manual. International Air Transport Association, Montréal, Quebec, 2017. ISBN 978-929229-505-9. 